\title{
Geological applications using geospatial standards - an example from OneGeology-Europe and GeoSciML
}

\author{
John Laxton ${ }^{1}$, Jean-Jacques Serrano ${ }^{2}$, Agnes Tellez-Arenas ${ }^{2}$ \\ ${ }^{1}$ British Geological Survey, Edinburgh, United Kingdom \\ ${ }^{2}$ BRGM, Orleans, France
}

\begin{abstract}
GeoSciML has been developed to enable the interchange of geoscience information, principally that portrayed on geological maps as well as boreholes. A GeoSciML testbed was developed both to test the implementation of the data model and its application in web services. The OneGeology-Europe project aims to use the GeoSciML data model, and build on the experience of the GeoSciML testbed, in implementing a geoportal for a harmonised geological map of Europe at 1:1 million scale. This involves the integration of web services from twenty participating organisations. An important objective of OneGeology-Europe is to contribute to INSPIRE, both through the development of a geological data specification and the use of the INSPIRE technical architecture. GeoSciML and the OneGeology-Europe project are also steps towards incorporating geoscience data into a Digital Earth. Both the development of GeoSciML, and the implementation of web services for GeoSciML and OneGeology-Europe, have followed a standards based methodology. The technical architecture comprises a geoportal providing access to a CSW catalogue service for metadata describing both the data and services available. OneGeology-Europe will provide both WMS view and WFS download services, which aim to be compliant with the INSPIRE implementing rules.
\end{abstract}

Keywords: data exchange model, web services, spatial data standards, INSPIRE, digital earth

\section{Introduction}

The paper will describe the development of GeoSciML (Geoscience Mark-up

Language) as an interoperability standard for geoscience information and its use in the

OneGeology-Europe project. In particular we will show how both development and

implementation have made full use of international geospatial information standards.

Interoperability is a key element in enabling the Digital Earth vision and the adoption

of international standards is an essential component in achieving interoperability

(Nativi, S \& Domenico, B, 2009). The development of GeoSciML and its use in the

OneGeology-Europe project can therefore be seen as important steps towards bringing geoscience information into a future Digital Earth. 


\subsection{Background to GeoSciML}

It is becoming increasingly important to query and exchange geological information between geological data providers for legal, social, environmental and geoscientific reasons. Coincidentally, technological opportunities arising from the evolution of geospatial and geological information standards are making interoperability a viable endeavour.

In recognition of these trends an Interoperability Working Group (IWG) (CGI, 2009) was formed under the auspices of the International Union of Geosciences (IUGS) Commission for the Management and Application of Geoscience Information (CGI), with the aim of developing relevant and timely standards and testing them within the context of individual data providers, mainly geological surveys. The development of GeoSciML has been a global initiative with active participants in the CGI Interoperability Working Group drawn from Europe, North America, Asia and Australia. The ultimate objective of the working group is to enable seamless web integration of select geoscience information hosted by different data providers in varied formats.

More specific objectives (CGI, 2009) are:

- To develop a conceptual model of geoscientific information drawing on existing data models,

- To implement an agreed subset of this model in an agreed schema language,

- To implement an XML/GML ${ }^{1}$ encoding of the model subset. GML is the grammar defined by the Open Geospatial Consortium (OGC) to express geographical features,

- To develop a testbed to illustrate the potential of the data model for interchange,

- To identify areas that require standardised classifications in order to enable interchange.

The GeoSciML data model has been developed in $\mathrm{UML}^{2}$ from which a schema has been generated automatically. In order to test the model a series of use-

\footnotetext{
${ }^{1}$ XML : eXtended Markup Language ; GML : Geography Markup Language

${ }^{2}$ UML : Unified Modeling Language
} 
cases were developed which were then implemented in web services by the GeoSciML partner organisations. All GeoSciML materials, including the GeoSciML UML model, XML/GML schema, instance documents, and full documentation and 'cookbooks' to assist users with implementation are available from http://www.cgiiugs.org/tech_collaboration/geosciml.html.

The development of GeoSciML has been entirely funded by the participating organisations through the provision of staff time and the costs of travel and computing resources. This approach has the disadvantage that it has excluded some less well funded organisations, but has had the advantage that the development process has not been constrained by the timescale of a single project or the requirements of external funding organisations. GeoSciML development has been constrained instead by the actual use cases and requirements of the participating geological surveys who see GeoSciML as necessary to fulfil their role of disseminating geoscience information. This approach has enabled the IWG to make significant progress in the development of GeoSciML since it was formed in 2004.

\subsection{Background to OneGeology-Europe}

The OneGeology-Europe (EC, 2008) project is a collaboration of twenty-nine European organisations, comprising twenty geological surveys and nine academic and user organisations, and is co-funded by the European Union under the eContentplus programme. OneGeology-Europe has developed from the earlier OneGeology-Global initiative which aims to 'Make web-accessible the best available geological map data worldwide at a scale of about 1: 1 million, as a geological survey contribution to the International Year of Planet Earth.' (http://www.onegeology.org/home.html). OneGeology-Global currently has 113 countries participating and was set up with a Memorandum of Understanding (OneGeology, 2007) between the participating 
geological surveys and UNESCO, the Commission for the Geological Map of the

World (CGMW), the International Union of Geosciences (IUGS), the International

Year of Planet Earth (IYPE), and the International Steering Committee for Global

Mapping (IGSCGM). OneGeology-Global also uses GeoSciML.

The key objectives of OneGeology-Europe (EC, 2008) are to build on the work of

OneGeology-Global in Europe with the following actions:

- To bring together a web-accessible, interoperable geological spatial dataset for the whole of Europe at 1:1 million scale,

- To develop a harmonised specification for basic geological map data and make significant progress towards harmonising the dataset,

- To accelerate the development and deployment of a nascent international interchange standard for geological data - GeoSciML,

- To facilitate re-use and addition of value by a wide spectrum of users and identify, document and disseminate strategies for the reduction of technical and business barriers to re-use,

- To address the multilingual aspects of access through a multilingual discovery portal,

- To move geological knowledge closer to the end-user.

In order to meet these objectives the project has identified the following key

deliverables:

- Use cases and best practice in meeting stakeholder needs,

- 1:1 million pan-European geological data specification, identification and sourcing,

- Data inventory and multilingual discovery metadata catalogue,

- Informatics specification, data model, interoperability and standards,

- Web portal and Registry development and implementation,

- Access and licensing protocols,

- Communication, dissemination and awareness,

- Access to high resolution geological \& applied datasets in national \& cross border situations,

- Liaison with related groups and initiatives.

It has been decided that the OneGeology-Europe project will use the

GeoSciML data model and build on the experience gained in developing the

GeoSciML testbed. It is also an objective of the OneGeology-Europe project to 
'contribute to INSPIRE' ${ }^{3}$. INSPIRE is an initiative of the European Community designed to create a Spatial Data Infrastructure (SDI) for Europe, and in particular to enable the sharing of environmental spatial information among public sector organisations and better facilitate public access to spatial information across Europe. More information about INSPIRE is available at http://inspire.jrc.ec.europa.eu/index.cfm. OneGeology-Europe aims to contribute to INSPIRE in the following ways:

- Use of ISO 19115/19119 for services metadata,

- Contributing to the INSPIRE geology data specification through use of the GeoSciML data model,

- Implementation of discovery, view and download services in line with INSPIRE requirements,

- Analysis of the data sharing practices of geological surveys across the European Union,

- Implementation of web service monitoring.

To ensure that the spatial data infrastructures of the countries of the European Union are compatible INSPIRE requires that common Implementing Rules (IR) are adopted in a number of specific areas including metadata (INSPIRE, 2009 (1)), discovery services (INSPIRE, 2009 (2)), view services, (INSPIRE, 2009 (3)), and download services (INSPIRE, 2009 (4)). The general principle that has been adopted by OneGeology-Europe is to try to use the INSPIRE implementing rules as much as possible.

\section{GeoSciML data model}

\subsection{Scope and structure}

The GeoSciML data model is designed to assist interoperability through a standardisation of the data structure used in geoscience and is built on top of international standards for data language, such as GML and the $\mathrm{W}^{4} \mathrm{C}^{4}$ web systems

\footnotetext{
${ }^{3}$ INSPIRE: Infrastructure for Spatial Information in the European Community

${ }^{4}$ W3C: World Wide Web Consortium
} 
standards. The model draws on pre-existing geoscience data models, in particular the North Amercian Data Model (NADM) (North American Geologic Map Data Model Steering Committee, 2004). The scope of the content of GeoSciML has been restricted in the current version to those geoscience objects which form the main components of a geological map: geological units; geologic structures; and earth materials. It also includes boreholes. In modelling the geological map the concept of the Geologic Feature, which can be either a Geologic Unit or a Geologic Structure, is central.

An important aspect of the model is that it separates the concept of the Geologic Feature, which can be considered a real world object instance the full spatial extent of which is unknown, from its occurrences which are termed Mapped Features. A typical geological map is made up of Mapped Features which are in turn specified by Geologic Features (which are often described in more detail in the map key or a related stratigraphic lexicon). A single Mapped Feature can be specified by only one Geologic Feature, but a Geologic Feature can have several Mapped Feature occurrences, on maps of different scales or in a 3D model for example. The main function of a Mapped Feature therefore is to hold geometry, which it inherits from standard types defined in GML (ISO/TC 211, 2007 (1)). This pattern contrasts with that of many GIS formats, such as ESRI shapefiles (ESRI, 1998), where geometry and attribution are tightly bound.

The GeoSciML Boreholes and Observations package re-uses standard components from the OGC Observations and Measurements package (Open Geospatial Consortium, 2007 (1); Open Geospatial Consortium, 2007 (2)). Boreholes are modelled as a special type of SamplingCurve feature but borehole logs can be described in two ways in the GeoSciML model - either as a series of discrete 
Observations down the borehole or as a series of log elements which associate to Mapped Intervals. Mapped Intervals are a type of Mapped Feature and in this approach a borehole can be considered as being akin to a linear geological map.

\subsection{Schematic and semantic interoperability}

The GeoSciML initiative will put in place the standards to enable the schematic interoperability of geoscience data, but semantic interoperability will only be achieved when the data content also conforms to agreed international standards. Figure 1 shows where GeoSciML fits within the hierarchy of interoperability. GeoSciML is useful even without full semantic interoperability as it enables Geological Survey Organisations (GSOs), and other geoscience data providers, to deliver the same type of information to customers in a standard format thus allowing easier analysis and processing.

The GeoSciML conceptual model assists in the development of semantic interoperability by identifying those concepts that require standardisation for such interoperability. For example the model has a feature 'GeologicEvent' described by the three properties eventAge, eventEnvironment and eventProcess. The eventAge property can be encoded using values from the International Commission on Stratigraphy (ICS) stratigraphic time chart, produced from the geologic time scale (Gradstein et al, 2004). This is an example of an internationally agreed set of concept definitions, and has been implemented in GeoSciML. There is however no agreed set of concepts for event age or event process, and this is the case for most geoscience properties. The IWG has therefore set up a Concept Definitions task group to develop simple vocabularies of concepts for such properties, designed specifically for interoperability. It is recognised that these vocabularies are a temporary measure until 
full concept definitions have been developed by the relevant international geoscience communities.

\subsection{Use of standards}

A central principle in the development of GeoSciML has been to conform to standards based methodologies published by OGC and ISO. GeoSciML is formally specified by a UML model known as an ‘application schema’ as defined in ISO 19109 (ISO/TC 211, 2005 (1)). From this model an XML schema is generated which is conformant with Geography Markup Language (GML) encoding as defined in ISO 19136 (ISO/TC 211, 2007 (1)). GML in turn draws its geometry from the spatial schema defined in ISO 19107 (ISO/TC 211, 2003 (2)) while GeoSciML uses metadata specified by ISO 19115 (ISO/TC 211, 2003 (1)). For boreholes and outcrops GeoSciML draws heavily on the OGC Sampling Features Model (Open Geospatial Consortium, 2007 (2)) and the OGC Observation Model (Open Geospatial Consortium, 2007 (1)).

It follows from the use by GeoSciML of existing standards and schema that the CGI Interoperability Working Group is only responsible for maintaining the geology-specific components of GeoSciML. It is intended that GeoSciML will extend into other areas of geoscience as required, but only where these are not already being modelled by other interest groups. Where such related activities exist the aim will be to cooperate with them to assist them to use GeoSciML in their modelling. GWML ${ }^{5}$ (Boisvert, E., Brodaric, B., 2007) is an example of a derived implementation of GeoSciML for groundwater. It is also the first official collaboration between GeoSciML and an external exchange model group. Mineral Occurrences (Seymon, A. et al, 2007) is an example of an inherited implementation of GeoSciML. It is being

\footnotetext{
${ }^{5}$ GWML: Groundwater Mark-up Language
} 
developed by the Australian Government Geologists Information Committee (GGIC) as a model to deliver mineral occurrences information as $\mathrm{WMS}^{6}$ and $\mathrm{WFS}^{7}$.

Australian State, territory and federal organizations presently govern the model.

\section{Web services standards and implementation}

\subsection{Requirements}

\subsubsection{GeoSciML testbed}

The results of the GeoSciML testbed

(http://appgeosciml.brgm.fr/GeoSciMLWeb/) were presented in the $33^{\text {rd }}$ IUGS

Meeting (Oslo, August 2008). The four main requirements of this testbed were:

- To implement a Metadata catalogue providing a Catalog Service for the Web (CSW) (Open Geospatial Consortium, 2007 (3)) to describe both services and datasets. The Metadata profile used are the standards ISO19115 (ISO/TC 211, 2003 (1)) and ISO19119 (ISO/TC 211, 2005 (2)),

- To implement WMS and WFS services. The WFS services should deliver a common GeoSciML based data model and be able to manage a specific list of OGC Filters,

- To implement web services to manage GeoSciML vocabularies,

- To develop a web client application demonstrating the use of the CSW, WFS and vocabularies services.

\subsubsection{OneGeology-Europe}

A main objective of the OneGeology-Europe project is to allow substantial progress towards INSPIRE goals: final users of the system specified and implemented by the project will be able to discover, view and download geoscience data across the

European Union (EU). From this major objective come several requirements:

- To define and implement a multilingual metadata profile for the geosciences, exploiting, extending and bringing up to date an existing Multilingual Thesaurus for the geosciences,

- To implement a Metadata catalogue with its metadata editor to register metadata of services and datasets, accessible through a multilingual discovery component (CSW 2.0.2) within the project data portal,

- To define a common Data Model based on GeoSciML to exchange geological data. Each data provider has to map its data to this common language,

\footnotetext{
${ }^{6}$ WMS: Web Map Services

${ }^{7}$ WFS: Web Feature Services
} 
- To define and implement a set of View and Download services for delivering and processing the geological data,

- To implement a geoportal to provide the user with a user-friendly way to discover, view and access geological data.

\subsection{Architecture}

In both the GeoSciML testbed and OneGeology-Europe the architecture is based on the INSPIRE technical architecture, implementing Network services (INSPIRE, 2009 (2), INSPIRE, 2009 (3), INSPIRE, 2009 (4)). This architecture addresses the "publish-find-bind” pattern, completed by the "agree” process as INSPIRE has also to deal with rights management. Each country involved in the project (fewer than ten in the GeoSciML testbed, twenty in OneGeology-Europe), implement view and download services. Each service dataset is described by a metadata record conforming to the metadata profile, in the Metadata catalogue (this implements the "publish" process). A search engine included in the geoportal allows the end-user to discover these services and datasets (this implements the "find" process). This search engine is a web client using the CSW service over the metadata catalogue. The geoportal then directly accesses the services hosted by the countries to display or access the datasets (this implements the "bind” process, the "agree” process is not addressed in the OneGeology-Europe and GeoSciML testbed projects). Figure 2 gives an overview of the OneGeology-Europe architecture.

\subsection{Metadata}

The Multilingual Geological Metadata Profile currently developed in the OneGeology-Europe project is based on ISO 19115 (ISO/TC 211, 2003 (1)), ISO 19119 (ISO/TC 211, 2005 (2)), and ISO19139 (ISO/TC 211, 2007 (2)) (for XML encoding). This profile takes into account INSPIRE Implementing Rules for metadata (INSPIRE, 2009 (1)). Since the OneGeology-Europe catalogue will harvest several 
national catalogues to get the records, the OneGeology-Europe profile will not contain any extension of the ISO standard.

Regarding multilingualism, the title, abstract, lineage statement, organization data (organization name, address, country), and conditions for access will be translated into at least eight languages. The code-lists, either ISO or developed by the project, such as keywords and some quality information, will also be translated.

An important part of the metadata profile concerns quality information. Harmonization of practices over Europe is a challenge for this topic and is still in progress, but it should follow several rules:

- Lineage should contain both the process and the source material, using some structured rules already existing in several countries,

- Harmonization method and level of harmonization (semantic (internal or external classification) and geometry) should be described.

To harmonize as far as possible the quality data in use in the twenty countries some templates are defined for the free text quality fields. The aim is not to obtain, automatically, formally structured fields, but rather more readable and unified texts.

\subsection{Catalogue service}

To manage the metadata records and to enable searching for references to available geological datasets and services in both projects, a catalogue client and an associated catalogue service is set up. It is based on the OGC standard Catalogue Service for the Web (CSW) (Open Geospatial Consortium, 2007 (3)), with an ISO Application Profile. Any CSW client is able to use this interface to access the catalogue.

\subsubsection{The catalogue in the GeoSciML testbed}

The catalogue is based on GeoNetwork, modified to introduce some new functionality (to create automatically service metadata records from the service capabilities, to create also automatically a dataset metadata record from the layer information available in the WMS capabilities, and to create the link between service metadata 
and related datasets metadata). GeoNetwork is a standards based, free and opensource catalogue application to manage spatially referenced resources through the web (GeoNetwork, 2009). It stores not only services and datasets but also OGC SLD ${ }^{8}$ (Open Geospatial Consortium, 2005 (1)) files (used with WMS 1.1 (Open Geospatial Consortium, 2006 (1))) providing some common portrayal rules for the GeoSciML vocabularies such as the lithology. The services offered are:

- Manual creation and editing of the records,

- Import and export of records in ISO XML format,

- Harvest services (WFS and WMS) for creating services and associated datasets.

The GetCapabilites, GetRecords and GetRecordsByID operations are implemented.

\subsubsection{The catalogue in the OneGeology-Europe project}

This catalogue is MIcKA, the software used by the Czech Geological survey. It provides the usual manual creation and editing of the records, and implements the following CSW operations:

- GetCapabilities, GetRecord and GetRecordById. The GetRecord operation manages all the filters required by the INSPIRE implementing rules regarding the discovery service (INSPIRE, 2009 (2)),

- Harvesting of services (WFS and WMS) for creating services and datasets,

- Harvesting other CSW services to import services and datasets from national catalogues.

Figure 3 shows the web interface of the OneGeology-Europe Metadata catalogue for the addition of a record

\subsection{View and download data service}

Once the user has discovered datasets relevant to his purpose, web services are provided to view and download the data. These web services are based on ISO and OGC standards: Web Map Service (WMS) (Open Geospatial Consortium, 2006 (2)) for the view service and Web Feature Service (WFS) (Open Geospatial Consortium,

\footnotetext{
${ }^{8}$ SLD: Styled Layer Descriptor
} 
2005 (2)) for the data download service. For their implementation, OneGeology-

Europe takes into account, as much as possible, rules defined by the INSPIRE

Implementing Rules for view services (INSPIRE, 2009 (3) and download services

(INSPIRE, 2009 (4).

\subsubsection{View services}

The view services are implemented by OGC Web Map Services, with some specific requirements to be, as far as possible, INSPIRE compliant (mainly the use of WMS 1.3; a 'Language' parameter to provide layer title, abstract, and keywords and to deliver legend in the requested language; and some INSPIRE mandatory performance requirements which could be addressed by the OneGeology-Europe project). Other requirements come from the use cases of the project. The rules are the following:

- WMS required are: OGC WMS1.3 with SLD WMS profile 1.1.0,

- A new parameter "language" must be managed by each operation,

- The GetCapabilities, delivering the metadata of the service and the datasets is mandatory and should be translated into at least the national language of the dataset provider and English (one GetCapabilities file per language). The language parameter allows software using the WMS to access the translated GetCapabilities response. In the GetCapabilities response some fields are mandatory and should be conformant with the Metadata profile,

- The GetFeatureInfo operation is required, and the delivery format is harmonized:

o Text/html should follow a template and should be (as far as possible) translated in accordance with the language parameter.

o Text/xml response must deliver GeoSciML Data Model (harmonized data model),

- The GetMap operation should be able to manage a SLD_BODY and SLD parameter respecting the GeoSciML Data Model (name of the attributes), using ogc:filter such as in the following example:

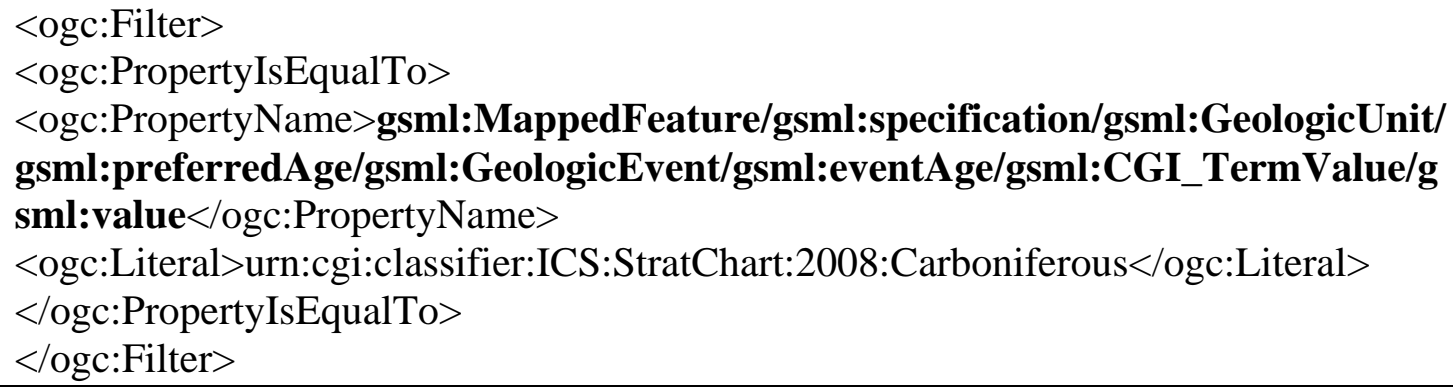


The name of the datasets should be harmonized in order to guarantee to the system using them that they implement the same data model. A client application (for instance, a geoportal) can then use the CSW service of the catalogue to find all the datasets with a specific harmonized name. We also use keywords to indicate resources compliant with some specifications defined by a community; this option is to solve the issue of lack of classification possibilities in the catalogue.

The aim of these rules is to fill two main requirements. The first is multilingualism, for instance to have two different GetCapabilities responses for two languages allowing us to provide two different legend URLs, one for each language. The second aim is to enable us to provide harmonized views of the datasets, both for the image (GetMap) and the attributes (GetFeatureInfo).

\subsubsection{Download data service}

The Download data service is implemented by an OGC Web Feature Service. This service is still under discussion in the OneGeology-Europe project, so only some preliminary rules are given here. As for the view service, the OneGeology-Europe project will try to use INSPIRE rules for the download service. INSPIRE recommends the provision of two types of download service:

- Download pre-defined data stored in a file using a simple HTTP Get protocol (the link is defined into the dataset metadata),

- Direct access to data, with the possibility of using search criteria (bounding box, values of selected attributes, ...). This type of download will be based on ISO standards WFS 2.0 (ISO/TC 211, 2009 (1)) and Filter Encoding (ISO/TC 211, 2009 (2)).

The Language parameter is also mandatory for INSPIRE.

The OneGeology-Europe project will implement the "direct access service” but with WFS 1.1.0 (as there is not yet stable software implementing WFS 2.0), and with the Filter Encoding standard. 
A key objective is that the GetFeature operation delivering the data should deliver the GeoSciML schema. A specific list of ogc:filter should be defined (and was defined for the GeoSciML testbed).

\section{Example: query all the geologic units where the lower age is "Middle}

\section{Jurassic":}

$<$ ogc:Filter $>$

$<$ ogc:PropertyIsEqualTo $>$

< ogc:PropertyName>gsml:MappedFeature/gsml:specification/gsml:GeologicUnit/gs ml:preferredAge/gsml:GeologicEvent/gsml:eventAge/gsml:CGI_TermRange/gsml:lo wer/gsml:CGI_TermValue/gsml:value</ogc:PropertyName>

$<$ ogc:Literal>urn:cgi:classifier:ICS:StratChart:2008:MiddleJurassic</ogc:Literal> $</$ ogc:PropertyIsEqualTo $>$

$</$ ogc:Filter $>$

\section{Example: query all the Dykes:}

$<$ ogc:Filter $>$

$<$ ogc:and $>$

$<$ ogc:PropertyIsEqualTo $>$

< ogc:PropertyName>gsml:MappedFeature/gsml:specification/gsml:GeologicUnit/gs ml:bodyMorphology/gsml:CGI_TermValue/gsml:value</ogc:PropertyName>

< ogc:Literal>urn:cgi:classifier:CGI:GeologicUnitMorphology:200811:dyke</ogc:Lit eral $>$

$</$ ogc:PropertyIsEqualTo $>$

$<$ ogc:and $>$

$</$ ogc:Filter $>$

In the OneGeology-Europe project, a language parameter is added in the same way as for the view service (Web Map Service).

\subsubsection{The language parameter}

Managing multilinguality is a key point for European Geological Surveys in the

INSPIRE context. As the standards used for discovery, view, and download services

(CSW, WMS, WFS) do not yet address this issue, no software is available. The

solution implemented by OneGeology-Europe is to provide a software component

(the 1GE-Connector) on top of these standard services to select which service to request according to the requested language. 


\section{Benefits of web services standards and harmonization: examples of usage}

\subsection{First example: use of the common data model and SLD-profile in a WMS}

The OneGeology-Europe geoportal (http://onegeology-

europe.brgm.fr/geoportal/viewer.jsp) will display a harmonized geological dataset of the whole Europe. Figure 4 is a screenshot of the OneGeology-Europe portal showing a portion of the harmonized geological map of Europe at 1:1 million scale. This dataset is technically composed of twenty images requested from twenty Web Map Services. For this dataset, the geoportal provides a "query then view" function, using the SLD parameter of the GetMap operation. The user chooses some criteria, for example an age presented as a list of values coming from the OneGeology-Europe GeoSciML vocabularies. He also chooses a colour, and then applies the style.

In response to this action, the geoportal builds a SLD defining portrayal rules according to the ogc:filter using the GeoSciML name of the attributes. In the case of our example, age could be translated by the following value:

"gsml:MappedFeature/gsml:specification/gsml:GeologicUnit/gsml:preferredA ge/gsml:GeologicEvent/gsml:eventAge/gsml:CGI_TermValue/gsml:value”

Then a new GetMap request is sent with this SLD and each Web Map Service displays its part of the full dataset, using the same portrayal rules. The result is a map highlighting all the features having the same age.

Three standards are required to be able to provide such a function:

- $\quad$ OGC Web Map Services for the GetMap request,

- All the Web Map Services implementing (at least partially) the OGC SLD profile,

- A common standard data model (in our case, GeoSciML) applied at two levels: a common structure (names of the attributes), and a harmonized content (use of referenced and translated vocabularies).

Figure 5 shows several European countries hosting a WMS delivering the harmonized geological map of Europe at 1:1 million scale. Using the common data 
model (GeoSciML) a common SLD is sent to each WMS (request GetMap), to display all the geologic units of "proterozoic” age in pink.

\subsection{Second example: use of the common data model in a WFS}

One major challenge of the OneGeology-Europe project is to move geological knowledge closer to the end-user. In this context, a principal requirement is the legend of the dataset. It should be "easy to read" for a non-geologist, with details and some links to glossaries, but it should also be detailed and scientifically useful for a geologist.

The OneGeology-Europe portal provides a tool to display the legend of a small part of the map selected by the user. The geoportal requests all the Web Feature Services with an ogc:filter on the bounding box corresponding to the area. Then, it analyses the result to determine the list of the differing features to present either as a very detailed response, or as a basic legend of the features displayed. This tool also presents some statistics.

\subsection{Impact of OneGeology-Europe and GeoSciML on other projects}

The work done by Geological Surveys on GeoSciML has been used in the OneGeology-Europe project and in OneGeology-Global. The aim of OneGeologyGlobal is to create dynamic geological map data of the world available via the web (113 countries involved, about 60 WMS services available). There are less technical constraints for the providers than for European providers in the OneGeology-Europe project. A “simple” WMS is required with some metadata, and a legend, with naming rules (for services and layers). 
GEOSS $^{9}$ and INSPIRE share the same architecture principles, and the catalogue of services available for OneGeology is now registered in the GEOSS Component and Service Registry.

\section{Conclusions}

GeoSciML has been developed to enable interoperability of geoscience information. It is the product of an international collaboration between geoscience data providers, mainly geological surveys, and the self-funded nature of the activity is one of the factors that has enabled significant progress to be made. The development has followed a standards-based approach from the development of the data model through to the implementation of the schema derived from it. This has reduced development time through the re-use of pre-existing, and standard, elements allowing the GeoSciML development to concentrate on the specifically geoscientific components. Use of international spatial standards has also assisted in achieving interoperability, not just within the geoscience community but to other domains, thus enabling geoscience data to be incorporated into non-geoscientific applications.

GeoSciML enables schematic interoperability, but semantic interoperability requires agreement on the concepts used to describe geoscience information. Existing internationally agreed concepts, such as the age periods defined in the ICS Stratigraphic Time Chart, are used where these are available. For many geoscience concepts no such agreement exists however and the IWG has set up a Concept Definition task group to agree concepts for interoperability.

The OneGeology-Europe project is using GeoSciML in the web service delivery of a harmonized geological map of Europe at approximately 1:1 million scale. The implementation of web services using GeoSciML, both in the GeoSciML

\footnotetext{
${ }^{9}$ GEOSS: Global Earth Observation System of Systems (http://www.earthobservations.org/gci_gci.shtml)
} 
testbed and the OneGeology-Europe project, also follows a standards based methodology. This is necessary to ensure interoperable services, and in the case of OneGeology-Europe it is also providing a test of the implementation of the INSPIRE technical architecture which in turn is based largely on international standards.

A metadata catalogue of both data sets and services has been set up for OneGeology-Europe along with a catalogue service, conforming to the OGC CSW standard, to manage and access it. In addition OneGeology-Europe has set up view and download services which aim to be INSPIRE compliant as far as possible and use the OGC WMS and WFS standards.

The use of GeoSciML and OGC standards enables a user of the OneGeologyEurope portal to query the twenty component Web Map Services using a common vocabulary and display the results in a common style. The use of the OGC WFS standard in conjunction with GeoSciML enables the production and display of a detailed legend of a user selected area, drawn from one or more web services. It can be seen therefore that the schematic and semantic standardisation of the data model in GeoSciML, accompanied by the use of OGC web service standards, has enabled a significant level of geoscience data interoperability. The experience that has been gained in this work will be relevant to the application of INSPIRE standards to geoscience and to incorporating geoscience data into a future Digital Earth. ACKNOWLEDGEMENTS The development of GeoSciML was carried out by an international group of collaborators under the CGI Interoperability Working Group (https://www.seegrid.csiro.au/twiki/bin/view/CGIModel/InteroperabilityWG), and we have drawn on their work in describing GeoSciML. This paper is published by permission of the Executive Director of the British Geological Survey.

Figure legend 
Figure 1: The level of interoperability that GeoSciML is designed to address Figure 2: OneGeology-Europe Architecture overview

Figure 3: Web interface of the OneGeology-Europe Metadata catalogue: addition of a record

Figure4: OneGeology-Europe portal with the harmonized geological map of Europe Figure 5: Interoperability used to display only some geologic units.

Notes on contributors

John Laxton is a geologist at BGS who has over thirty years experience in applying information technology to the analysis and dissemination of geoscience information. He chairs the Interoperability Working Group which has been responsible for the development of GeoSciML and is a member of the design task group responsible for the GeoSciML data model. He is a member of the OneGeologyEurope project team responsible for developing the project's informatics specification and standards.

Jean-Jacques Serrano is a geoscience information expert at BRGM who has direct experience of INSPIRE. He is part of the 'Implementing Rules' drafting team and he also chairs the 'Network services’ drafting team. He participated in the European ORCHESTRA project to design an openservice-oriented architecture for risk management in Europe. He is a member of the CGI Interoperability Working Group developing and testing the data model for GeoSciML. He is involved in several European projects to define the architecture, to design data models and to select the standards to use.

Agnès Tellez-Arenas is a software engineer at BRGM involved in several projects to define and implement interoperable web services and web applications using them. She is in charge of the development of geoportals in various projects including Onegeology.

\section{References}

Boisvert, E., Brodaric, B., 2007, GroundWater Markup Language (GWML): Extending GeoSciML for Groundwater. American Geophysical Union, Fall Meeting 2007, abstract \#IN53C-03

CGI, 2009, Interoperability Working Group, http://www.cgiiugs.org/tech_collaboration/interoperability_working_group.html.

EC, 2008, eContentplus OneGeology-Europe project, http://ec.europa.eu/information_society/activities/econtentplus/projects/geo/on egeologyeurope/index_en.htm.

ESRI, 1998, ESRI Shapefile Technical Description, ESRI White Paper, p. 30. Available from http://www.esri.com/library/whitepapers/pdfs/shapefile.pdf.

GeoNetwork, 2009, GeoNetwork OpenSource. The complete manual, p. 146. Available from http://geonetworkopensource.org/documentation/manual/geonetwork-manual/Manual.pdf.

Gradstein, F.M., Ogg, J.G., Smith, A.G, 2004, A geologic time scale: Cambridge, Cambridge University Press, p. 589 
INSPIRE, 2009 (1), INSPIRE Metadata Implementing Rules: Technical Guidelines based on EN ISO 19115 and EN ISO 19119, p. 74. Available from http://inspire.jrc.ec.europa.eu/reports/ImplementingRules/metadata/MD_IR_a nd_ISO_20090218.pdf.

INSPIRE, 2009 (2), Technical Guidance for INSPIRE Discovery Services, p. 17. Available from http://inspire.jrc.ec.europa.eu/documents/Network_Services/Technical\%20Gui dance\%20Discovery\%20Services\%20v2.0.pdf.

INSPIRE, 2009(3), Technical Guidance to implement INSPIRE View Services, p. 36. Available from http://inspire.jrc.ec.europa.eu/documents/Network_Services/Technical\%20Gui dance\%20View\%20Services\%20v\%202.0.pdf.

INSPIRE, 2009 (4), Draft Technical Guidance for INSPIRE Download Services. p. 22. Available from http://inspire.jrc.ec.europa.eu/documents/Network_Services/INSPIRE\%20Dra ft\%20Technical\%20Guidance\%20Download\%20(Version\%202.0).pdf.

ISO/TC 211, 2003 (1), Geographic information - Metadata: ISO 19115.

ISO/TC 211, 2003 (2), Geographic information - Spatial schema: ISO 19107.

ISO/TC 211, 2005 (1), Geographic information - Rules for application schema: ISO 19109.

ISO/TC 211, 2005 (2), Geographic information - Services: ISO 19119.

ISO/TC 211, 2007 (1), Geographic information - Geography mark-up language (GML): ISO 19136.

ISO/TC 211, 2007 (2), Geographic information - Metadata - XML schema implementation: ISO 19139.

ISO/TC211, 2009 (1), Geographic information - Web Feature Service: ISO/DIS 19142

ISO/TC 211, 2009 (2), Geographic information - Filter encoding: ISO/DIS 19143

Nativi, S., and Domenico, B, 2009, Enabling interoperability for Digital Earth: Earth Science coverage access services. International Journal of Digital Earth, 2, 79104.

North American Geologic Map Data Model Steering Committee, 2004, NADM Conceptual Model 1.0 - A conceptual model for geologic map information, US Geological Survey Open-file Report 2004-1334, p. 58. Available from http://pubs.usgs.gov/of/2004/1334/2004-1334.pdf. 
OneGeology, 2007, A memorandum of understanding between UNESCO, CGMW, IUGS, IYPE, ISCGM and an international consortium of geological surveys to support global geoscience mapping and create an international coordinating committee. Available from http://www.onegeology.org/docs/MoU-signedJuly2007.pdf.

Open Geospatial Consortium Inc, 2005 (1), OpenGIS Styled Layer Descriptor Profile of the Web Map Service, project document 05-078r4

Open Geospatial Consortium Inc, 2005 (2), Web feature service (WFS) implementation standard, version 1.1, OGC project document 04-094.

Open Geospatial Consortium Inc, 2006 (1), Web map service (WMS) implementation standard, version 1.1.1, OGC project document 01-068r3

Open Geospatial Consortium Inc, 2006 (2), Web map service (WMS) implementation standard, version 1.3.0, OGC project document 06-042.

Open Geospatial Consortium Inc, 2007 (1), Observations and Measurements - Part 1 Observation schema, OGC project document 07-022r1.

Open Geospatial Consortium Inc, 2007 (2), Observations and Measurements - Part 2 Sampling Features (1.0), OGC project document 07-002r3.

Open Geospatial Consortium Inc, 2007 (3), OpenGIS Catalogue Service (CSW) Implementation Specification, OGC project document 07-006r1.

Seymon, A., Wyborn, L., Simons, B.,, Raymond, O., Andrews, G., Denaro, T., Jenkin, G., Lewis, P., Llorca, J., and McClennaghan, M, 2007, The Australian Mineral Occurrence data exchange model. Geoinformatics 2007 Conference, Paper No. 3-23. Available from http://gsa.confex.com/gsa/2007GE/finalprogram/abstract_122928.htm 
semantic

schematic

syntax

systems
Data content

Data structure

Data language

Data systems
Geoscience community

GeoSciML

\section{OpenGIS}

community (OGC) 


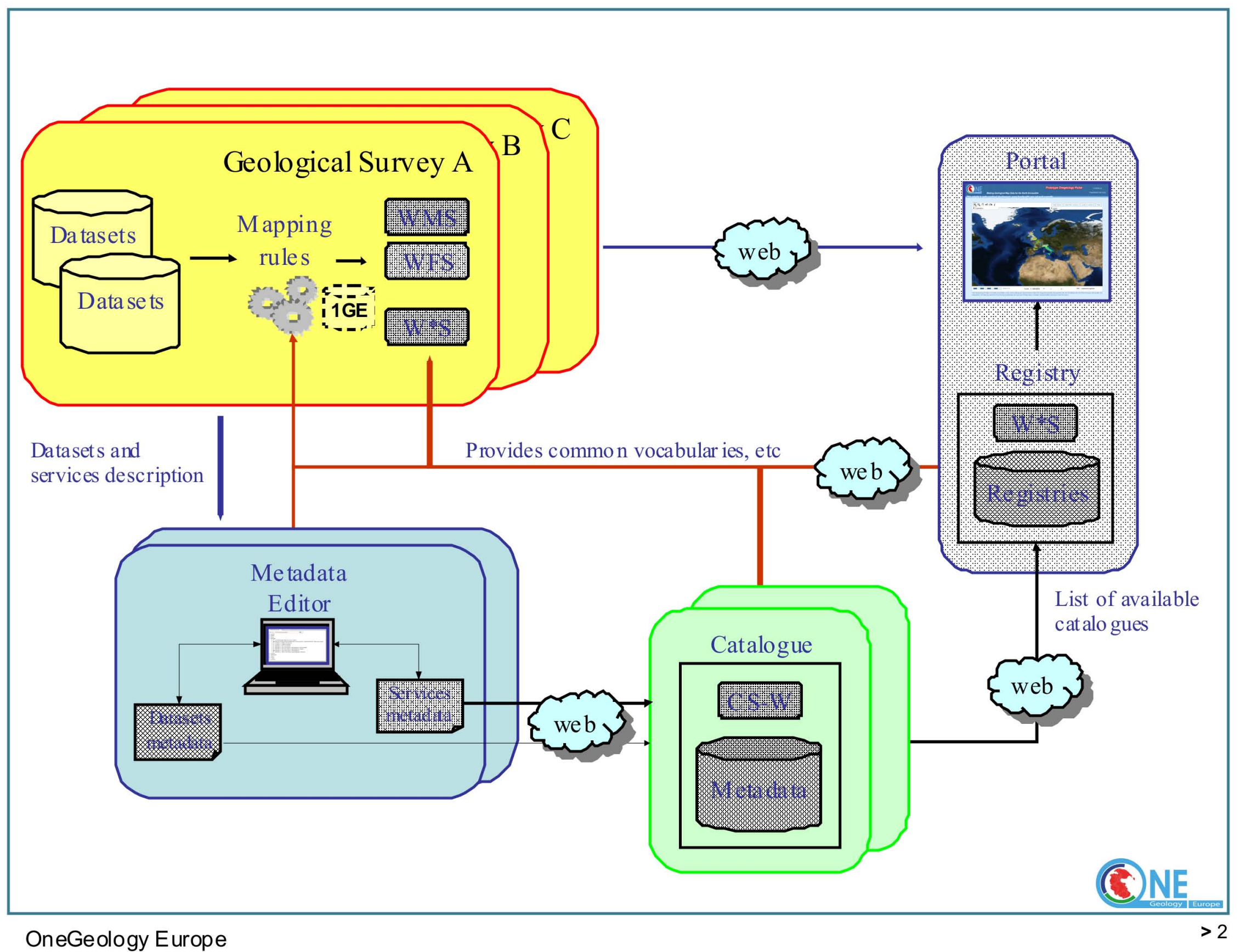




\begin{tabular}{l} 
Profile \\
oneGeology \\
Micka \\
ISO 19119 All \\
ISO 19119 handatory \\
ISO 19119 Core \\
DC / ISO 19119 \\
f Stop editing \\
की Same \\
\hline X Cancel edit \\
? Help \\
\hline (1) Info
\end{tabular}

\section{NEW RECORD / UPDATE RECORD}

@ ONEGEOLOGY-EUROPE - BRGM - France 50k - Vendee (WP9)

\section{Record administration}

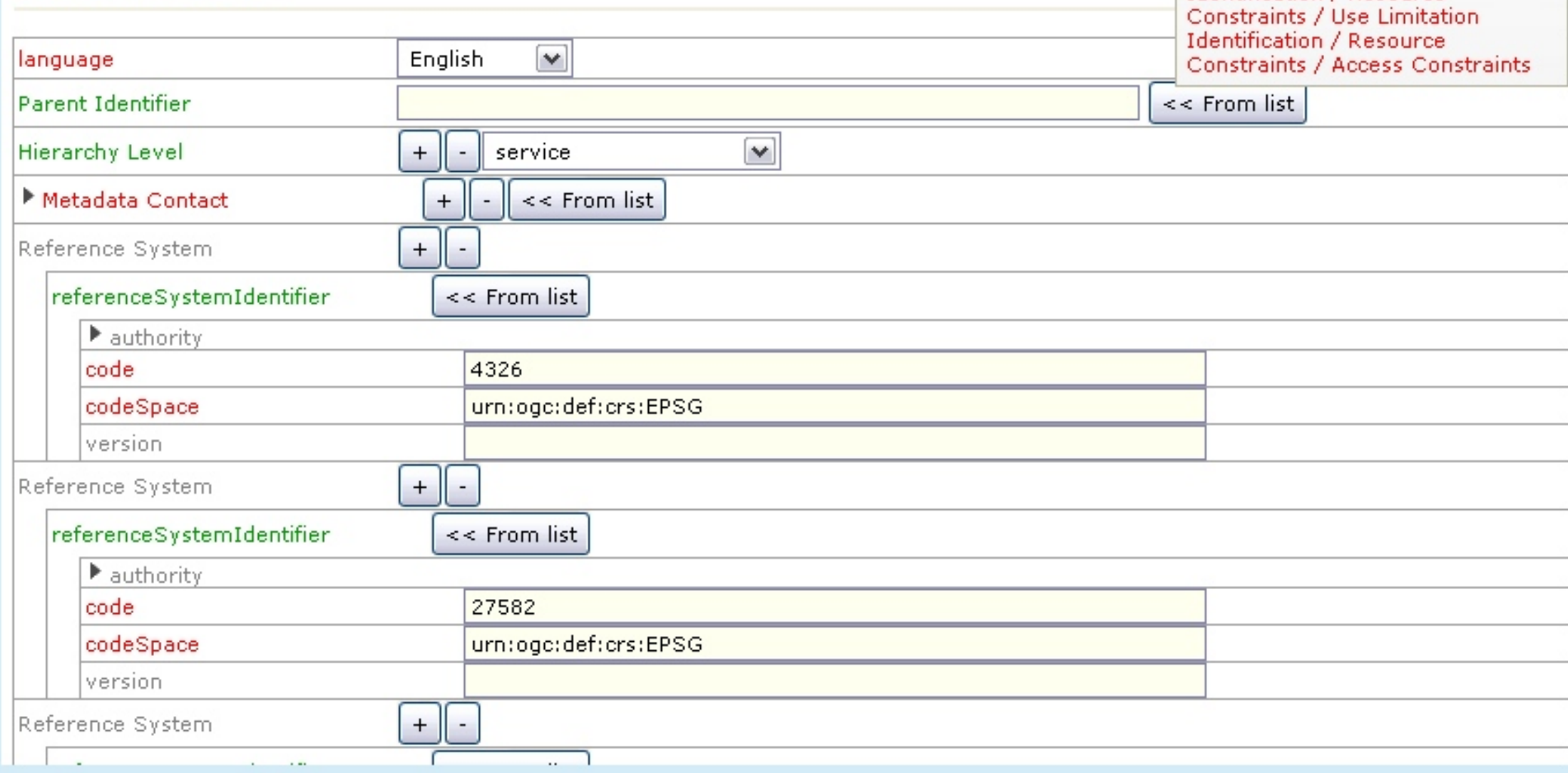

Identification / citation / Date

Identification / extent / Temporal Element Identification / Point of Contact /

role

Identification / Resource

Constraints / Access Constraint

人

S 
Layers

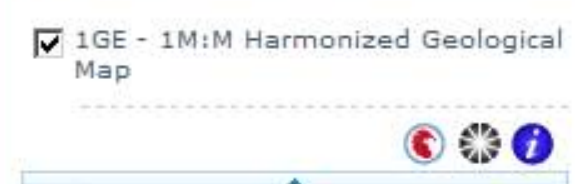

4t

Modify Opacity

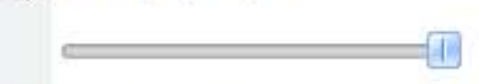

X Remove Layer

Thematic analysis

(9) View response times

\section{○Q}

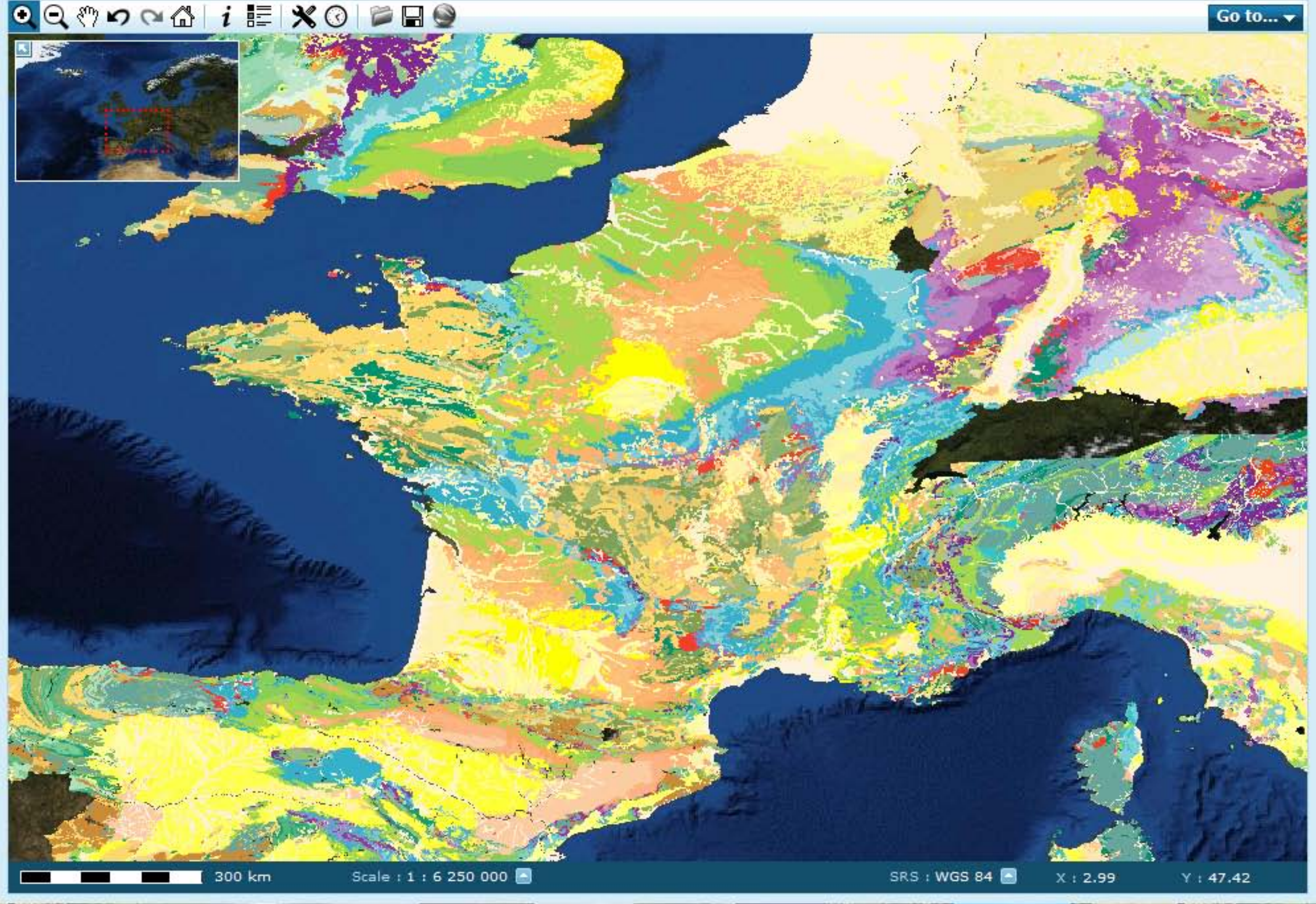




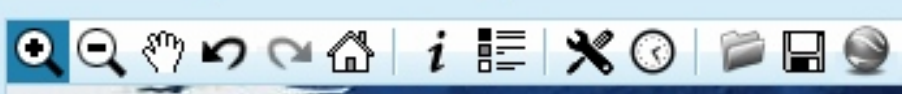

$\checkmark$ Thematic analysis

$x$

Warning: The $1 \mathrm{G}-\mathrm{E}$ profile is not yet defined, so the examples proposed bellow are not for scientific use.

GeoScimL services are under development, so this tool has some limited functions, checking too much acge is not recommanded.

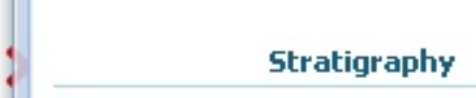

+- $\square$ Phanerozoic

फ-凹Proterozoic

句-Neoproterozoic

$\mapsto \checkmark$ Ediacaran

-øCryogenian

Tonian

비 Mesoproterozoic

ঋ Stenian

- Ectasian

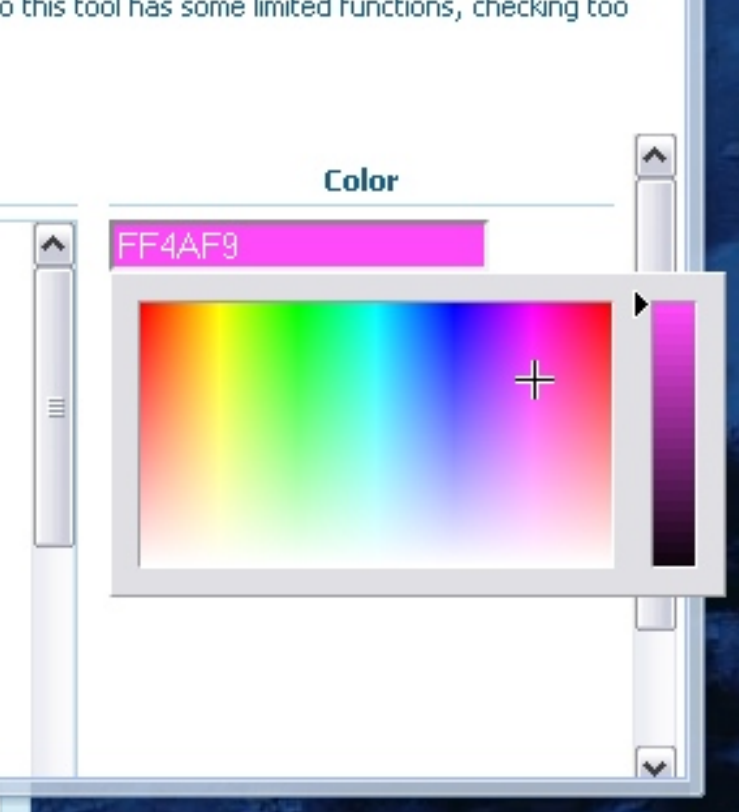

$\checkmark$

$\square \quad \square 1200 \mathrm{~km}$

Scale: 1:25000000 슨

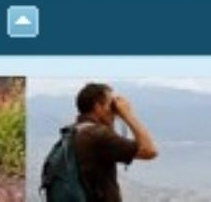

$x: 28.12$

Go to...

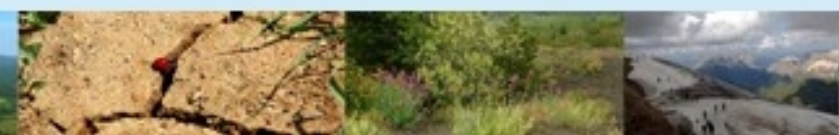

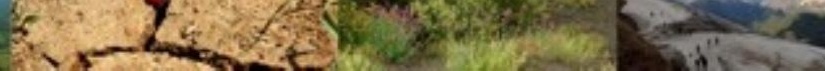

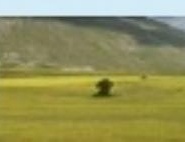

